\title{
Lumen
}

Selected Proceedings from the Canadian Society for Eighteenth-Century Studies

\section{Matérialité du texte et attentes de lecture. Concordances ou discordances?}

\section{Roger Chartier}

Volume 36, 2017

URI : https://id.erudit.org/iderudit/1037851ar

DOI : https://doi.org/10.7202/1037851ar

Aller au sommaire du numéro

Éditeur(s)

Canadian Society for Eighteenth-Century Studies / Société canadienne d'étude du dix-huitième siècle

ISSN

1209-3696 (imprimé)

1927-8284 (numérique)

Découvrir la revue

Citer cet article

Chartier, R. (2017). Matérialité du texte et attentes de lecture. Concordances ou discordances ? Lumen, 36, 1-20. https://doi.org/10.7202/1037851ar 


\title{
Matérialité du texte et attentes de lecture. Concordances ou discordances?
}

\author{
Roger Chartier \\ Collège de France et Université de Pennsylvanie
}

La réflexion que je voudrais proposer trouve son origine dans la confrontation de deux notions qui ont profondément transformé notre approche de la culture écrite et de la littérature. La première est celle de «matérialité du texte» telle qu'elle été définie dans un article devenu classique, «The Materiality of the Shakesperean Text», publié en 1993 par Margreta De Grazia et Peter Stallybrass dans Shakespeare Quarterly ${ }^{1}$. Leur cible était double. La critique visait, d'abord, contre les approches strictement formalistes, celles du «New Criticism» ou de la "Nouvelle critique ", qui considèrent les textes comme des structures linguistiques dont le fonctionnement est tenu comme tout à fait indépendant des modalités matérielles d'inscription de l'écrit. La seconde cible, plus récente alors, était le «New Historicism» qui historicise les relations ou «négociations» entre les discours et pratiques du monde social et les œuvres littéraires mais sans pour autant prendre en compte d'une historicité première : celle des formes de publication des textes eux-mêmes. De là, leur constat sans appel:

Les formalistes exigent une attention minutieuse aux plus petits détails linguistiques de la littérature sans accorder la moindre pensée aux pratiques extérieures à l'auteur et qui sont souvent à l'origine de ces mêmes détails. Les adeptes de l'historicisme, qui recherchent dans les œuvres de Shakespeare la trace des structures discursives de la fin du XVI et du

1. Margreta de Grazia et Peter Stallybrass, «The Materiality of the Shakesperean Text », Shakespeare Quarterly, 44, 3, automne 1993, p. 255-283. 
début du XVII ${ }^{e}$ siècle, oublient à quel point ces structures sont des constructions du XVIII siècle².

Le concept de «matérialité du texte» visait donc à surmonter l'opposition classique mais trompeuse entre, d'un côté, l'œuvre et, de l'autre, le livre ou l'objet imprimé. Cette distinction bien nette semblait définir des tâches très différentes: celles des historiens de la littérature, voués à l'étude de la genèse et des significations des œuvres, et celle des historiens du livre ou de l'édition, attachés à la compréhension des modalités de publication et circulation des textes. Classique, cette distinction a, paradoxalement peut-être, été renforcée, et non pas amoindrie, par l'érudition de la «New Bibliography» ou, comme l'on dit en français, la bibliographie matérielle qui analyse avec rigueur les différents états imprimés d'une même œuvre (éditions, émissions, exemplaires) pour neutraliser les corruptions infligées au texte par les pratiques de l'atelier typographique. Dans cette discipline, qui le plus souvent ne peut ou ne veut étudier que les objets imprimés qui ont transmis les textes, l'obsession majeure est pourtant celle de l'œuvre en son état originel, telle que son auteur l'a pensée, voulue, écrite ou dictée. De là, la distinction dans chaque texte imprimé entre «essentials » et «accidentals», entre les éléments consubstantiels à l'œuvre et les altérations qui relèvent des préférences ou des bévues des éditeurs, des correcteurs ou des compositeurs. De là, aussi, la quête de l'«ideal copy text ", du manuscrit écrit, dicté ou corrigé par l'auteur, un manuscrit à jamais absent pour les auteurs d'avant le XVIII siècle, mais possiblement imaginé à partir des états imprimés de l’œuvre.

La bibliographie matérielle n'est pas la seule discipline qui affirme une telle césure entre l'œuvre en son identité propre et sa corruption par sa mise en imprimé. Il en va ainsi de la pratique philologique lorsque, comme dans le cas du Lazarillo de Tormes, magistralement étudié et édité par Francisco Rico, elle montre l'imposition des habitudes propres à l'imprimé (page de titre, bois gravés, division en chapitres, épigraphes) sur un texte conçu comme une lettre appartenant au genre à la mode en Italie et en Espagne dans les années 1530-1540 des «carte messagiere », des lettres en vulgaire - en ce cas une lettre adressée par Lázaro (et non Lazarillo comme l’indique le titre) à un

2. Ibid., p. 256 (traduit en français par D. Lemonnier et F. Laroque dans Genesis, 7, 1995, p. 9-27). 
correspondant anonyme alors qu'il est devenu crieur public à Tolède. Composé comme une épître sans divisions, ayant sans doute circulé sous cette forme grâce à des copies manuscrites, le texte passa ensuite entre les mains d'un imprimeur qui le soumit aux règles ordinaires des livrets imprimés. Mais comme l'écrit Francisco Rico, cet éditeur du texte «por desgracia, apenas entendió nada», «malheureusement, il ne comprit rien »3. Avec le Lazarillo de Tormes, c'est d'une manière extrême que le livre a corrompu l'œuvre, en la soumettant à un traitement qui en transformait le genre, obligeant ainsi ses éditeurs modernes à la retrouver dans son premier état en effaçant les formes imposées par déformations de l'imprimé.

Mais doit-on se satisfaire de l'opposition tranchée entre l'œuvre en sa pureté essentielle et le livre qui, au mieux, ne ferait que la transmettre comme un véhicule inerte et, au pire, l'altère et la corrompt? Les métaphores anciennes désignaient cette différence entre œuvre et livre en utilisant la relation qui unit, dans l'être humain, l'âme et le corps. Alonso Víctor de Paredes, imprimeur à Madrid et Séville et auteur du premier manuel sur l'art d'imprimer en langue vulgaire, intitulé Institución del Arte de la Imprenta y Reglas generales para los componedores, composé autour de 1680, exprime avec force et subtilité la double nature du livre, comme objet et comme discours. Il inverse la métaphore classique qui décrit les corps et les visages comme des livres, et il tient le livre pour une créature humaine parce que, comme l'homme, il a un corps et une âme: «J'assimile un livre à la fabrication d'un homme, qui a une âme rationnelle, avec laquelle Notre Seigneur l'a créé avec toutes les grâces que sa Majesté Divine a voulu lui donner; et avec la même toute-puissance il a formé son corps élégant, beau et harmonieux ${ }^{4}$.»

Le livre doit être considéré comme une créature humaine, mais pour Paredes l'âme du livre n'est pas seulement le texte tel qu'il a été composé, dicté, imaginé par son créateur. Elle est ce texte donné dans une disposition adéquate, «una acertada disposición» : «un livre parfaitement achevé consiste en une bonne doctrine, présentée comme il

3. «Lázaro de Tormes », Lazarillo de Tormes, édition, étude et notes de Francisco Rico, Madrid, Real Academia Española, 2011, p. 96.

4. Alonso Víctor de Paredes, Institución y Origen del Arte de la Imprenta y Reglas generals para los componedores, éd. et prologue de Jaime Moll, Madrid, El Crotalón, 1984 , fol. 44 verso. 
le faut grâce à l'imprimeur et au correcteur, c'est cela que je tiens pour l'âme du livre; et c'est une bonne impression sur la presse, propre et soignée, qui fait que je peux le comparer à un corps gracieux et élégant ${ }^{5} »$. Si le «corps » du livre est le résultat du travail des pressiers, son «âme » n'est pas façonnée seulement par l'auteur, mais elle reçoit sa forme de tous ceux, maitre imprimeur, compositeurs et correcteurs, qui prennent soin de la ponctuation, de l'orthographe et de la mise en page. Paredes récuse ainsi par avance toute séparation entre la substance «essentielle» de l'œuvre, tenue pour toujours identique à ellemême, quelle que soit sa forme, et les variations «accidentelles» du texte, qui résultent des opérations dans l'atelier. Pour lui, homme de l'art typographique, la fabrique du livre est aussi fabrique de l'œuvre.

La mise en garde n'a pas été oubliée par la critique textuelle. La «sociologie des textes» telle que l'a définie D. F. McKenzie, a pour point de départ l'étude des modalités de publication, de dissémination et d'appropriation des textes ${ }^{6}$. Appuyée sur la tradition bibliographique, cette «sociologie des textes » met l'accent sur la matérialité du texte et l'historicité du lecteur avec une double intention: identifier les effets produits sur le statut, le classement et la perception des œuvres par les transformations de leur forme matérielle; montrer que les modalités propres de la publication des textes mettent en question la stabilité et la pertinence des catégories que la critique associe spontanément et anachroniquement à la «littérature »: ainsi les notions d'« œuvre», d'«auteur », d'« originalité », de «propriété littéraire».

C'est contre l'abstraction des discours que l'étude de la matérialité du texte, entendue au sens qu'ont donné à cette expression Peter Stallybrass et Margreta de Grazia, rappelle que la production, non pas seulement des livres, mais aussi celle des textes eux-mêmes, est un processus qui implique, au-delà du geste de l'écriture, différents moments, différentes techniques, différentes interventions. Les transactions entre les œuvres et le monde social ne consistent pas uniquement dans l'appropriation esthétique et symbolique d'objets ordinaires, de langages multiples, de pratiques rituelles ou quotidiennes comme

5. Idem.

6. D. F. McKenzie, Bibliography and the Sociology of Texts (The Panizzi Lectures, 1985), Londres, The British Library, 1986. (traduction française, D. F. McKenzie, La bibliographie et la sociologie des textes, préface de Roger Chartier, Paris, Éditions du Cercle de la Librairie, 1991). 
le veut (à juste titre) le «New Historicism». Elles concernent plus fondamentalement les relations multiples, mobiles, instables, nouées entre l'œuvre et ses différents textes. Ainsi, le processus de publication, quelle que soit sa modalité, ne sépare jamais la matérialité du texte de la textualité du livre.

Cette matérialité n'est pas seulement celle qui a retenu l'attention de la «New Bibliography», soucieuse d'une description formalisée des objets imprimés afin de reconstituer le processus de leur impression et désireuse de reconnaître, grâce à l'identification des habitudes, des préférences ou du matériel typographique des compositeurs qui ont composé les différentes formes ou feuilles du livre, les altérations qu'ils ont pu infliger à l'œuvre. La «matérialité du texte» s'attache, quant à elle, à la «fonction expressive » des modalités d'inscription du texte dans le livre: le format, la mise en page, les choix graphiques et orthographiques, la ponctuation. Ces décisions, quel qu'en soit le responsable, «font le texte » - au moins pour les lecteurs de l'édition où elles se rencontrent. C'est en cela que, selon D. F. McKenzie, «forms affect meaning».

La formule peut conduire à penser que les effets propres à l'invention de Gutenberg ne sont peut-être pas ceux qui ont été le plus souvent soulignés. Ils concernent, fondamentalement, les relations entre les œuvres en tant que textes et les formes de leur inscription typographique. Si le livre imprimé hérite bien des structures fondamentales du livre manuscrit (i.e. la distribution du texte entre les cahiers et feuillets propres au codex, quelle que soit la technique de sa production et reproduction), il propose des innovations qui modifient profondément le rapport du lecteur à l'écrit. Il en va ainsi, par exemple, des paratextes ou, plus exactement dans la terminologie morphologique de Gérard Genette, des péritextes qui composent le seuil du livre. Avec l'imprimé, ces préliminaires (un terme qu'il faudrait peut-être préférer) acquièrent une identité rendue immédiatement perceptible par les signatures particulières (italiques, voyelles tildées, symboles) qui caractérisent le ou les cahiers qui constituent les préliminaires, toujours imprimés (avec les tables et les index) après l'achèvement de l'impression du corps du livre et souvent rédigés par le libraire ou l'imprimeur même lorsqu'ils sont signés avec le nom de l'auteur. Les métaphores architecturales qui aux XVI ${ }^{\mathrm{e}}$ et XVII siècles désignent ces «porches » qui mènent à l'ouvrage proprement dit, trouvent une 
traduction matérielle dans la séparation typographiquement marquée entre l'œuvre et son «vestibule» (selon le mot de Borges).

Par ailleurs, le livre imprimé rend plus commun que le manuscrit le rassemblement dans un même volume des œuvres d'un même auteur. Certes, l'innovation n'est pas absolue puisque c'est à partir de la mi-XIV siècle que, pour certains écrivains écrivant en langue vulgaire, s'est affirmée la pratique de n'assembler dans un même volume que des textes dont ils étaient les auteurs. Comme l'a montré Armando Petrucci, le geste rompait avec la tradition dominante de l'âge du manuscrit, celle des miscellanées qui rassemblent des textes de genres, de dates et d'auteurs fort différents. Mais la pratique se fortifie avec l'imprimé. Le Folio de 1616, composé par Ben Jonson lui-même, ou celui de 1623, qui ne doit rien à Shakespeare mais tout à ses anciens camarades et aux «stationers » qui possédaient ou ont acheté les « rights in copy» de trente-six de ses pièces, sont des illustrations exemplaires du lien fortement noué entre la matérialité du livre imprimé et le concept d'œuvre assignée à un nom propre. Il est donc sûr que les formes typographiques affectent le sens.

Mais à qui le lecteur du passé devait-il imputer ces formes? Chaque tradition de la critique textuelle a privilégié l'un ou l'autre des acteurs engagés dans le processus de composition et de publication des textes à l'âge de ce que l'on peut appeler l'ancien régime typographique. Pour la bibliographie matérielle, les choix graphiques et orthographiques sont le fait des compositeurs. Les ouvriers typographes des ateliers anciens n'avaient pas tous la même manière d'orthographier les mots ou de marquer la ponctuation. De là, la récurrence régulière des mêmes graphies ou des mêmes usages des signes de ponctuation dans les différents cahiers d'un même ouvrage en fonction des préférences et des habitudes du compositeur qui a en composé les pages. C'est pourquoi les «spelling analysis», avec l'étude de la récurrence des caractères endommagés ou des ornements, ont permis d'attribuer la composition de telle ou telle page à tel ou tel compositeur et, ainsi, de reconstituer le processus même de fabrication du livre, soit seriatim (c'est-à-dire en suivant l'ordre du texte), soit par formes (i.e. c'est-à-dire en composant à la suite toutes les pages assemblées dans une même forme et imprimées sur le même côté d'une feuille d'imprimerie). Ce mode de composition permettait de commencer l'impression d'une feuille alors même que toutes les pages d'un même cahier n'avaient pas 
encore été composées mais il supposait, aussi, le calibrage préalable et aussi exact que possible de la copie manuscrite.

Dans une autre perspective, plus philologique, l'essentiel est ailleurs: dans la préparation du manuscrit pour la composition telle qu'elle est opérée par les «correcteurs » qui ajoutent capitales, accents et ponctuation, qui normalisent l'orthographe, qui fixent les conventions graphiques et qui, souvent, sont en charge de la correction des épreuves. S'ils restent le résultat du travail des ateliers, ces choix ne sont plus ici assignés seulement ou principalement aux compositeurs, mais aux humanistes (clercs, gradués des universités, maîtres d'école) employés par les libraires et les imprimeurs pour assurer la plus grande correction possible de leurs éditions. Paolo Trovato a rappelé combien il était important pour les éditeurs du Cinquecento d'insister sur la «correction», effective ou supposée, de leurs éditions, affirmée sur les pages de titre par l'expression «con ogni diligenza corretto ${ }^{7}$ ». D'où le rôle décisif des «correcteurs» dont les interventions se déploient à plusieurs moments du processus d'édition: de la préparation du manuscrit à la correction des épreuves, des corrections en cours de tirage, à partir de la révision des feuilles déjà imprimées, à l'établissement des errata, en leurs diverses formes - les corrections à la plume sur les exemplaires imprimées, les feuillets d'errata ajoutés à la fin du livre ou les invitations faites au lecteur pour qu'il corrige lui-même son propre exemplaire.

Entre les $\mathrm{XVI}^{\mathrm{e}}$ et $\mathrm{XVIII}^{\mathrm{e}}$ siècles, les textes soumis ainsi aux décisions des «correcteurs», intervenant comme «copy-editor» ou "proof-reader », appartiennent à différents répertoires: les textes classiques, grecs ou latins; les œuvres en langue vulgaire qui ont eu une circulation manuscrite et auxquelles l'imprimerie impose ses propres normes de présentation du texte et, dans certains cas, comme celui des éditions italiennes, une normalisation linguistique; enfin, les manuscrits des écrivains contemporains dont la fort médiocre lisibilité irritaient fort correcteurs et compositeurs.

Faut-il donc penser que les auteurs demeuraient étrangers aux décisions et gestes qui donnaient aux livres leur matérialité? Dans son Dictionnaire publié en 1690, Furetière indique à l'article «Virgule »:

7. Paolo Trovato, Con ogni diligenza corretto. La stampa e le revisioni editoriali dei testi letterari italiani (1470-1570), Ferrara, UnifePress, 2009. 
«L'exactitude de cet Autheur va jusques là qu'il prend soin des points et des virgules ${ }^{8} »$. Implicitement, l'exemple d'emploi renvoie au désintérêt ordinaire des auteurs pour la ponctuation, mais il signale toutefois qu'il est des auteurs attentifs à la ponctuation de leurs textes - et plus généralement à la matérialité de leur inscription. Ronsard ou La Bruyère sont des exemples de cette «conscience typographique» qui les fait contrôler l'édition de leurs œuvres pour que puissent être produits dans la lecture les effets attendus de l'usage de la ponctuation, de la présence des lettres capitales dont certains mots sont dotés ou encore des jeux avec les différents caractères typographiques.

Lattention donnée à la matérialité du texte met ainsi en évidence la fragilité de la distinction entre mise en texte et mise en livre ainsi que la porosité de la frontière qui les sépare. Comme le constate Malcolm Parkes «la ponctuation imprimée peut refléter celle de l'auteur, celle de qui a préparé la copie pour la presse, celle du compositeur, ou les trois ensemble ${ }^{9}$ - et l'on pourrait ajouter à la liste celle du lecteur qui souvent modifie la ponctuation du livre imprimé qu'il lit, soit de sa propre initiative et en fidélité avec l'usage ancien qui veut qu’un livre imprimé ne soit véritablement achevé qu’après l'intervention de plusieurs mains, soit parce qu'il y a été invité par l'imprimeur lui-même afin de corriger les erreurs introduites dans la composition imprimée.

La cause est donc entendue. La matérialité du texte, et pas seulement sa lettre, guide ou informe la lecture. C'est pourquoi la perspective de Don McKenzie tient l'histoire de la lecture comme centrale tant pour la critique textuelle que pour l'histoire du livre. L'idée qu'un texte n'accède véritablement à l'existence que si un lecteur se l'approprie a sous-tendu toutes les démarches (herméneutique, phénoménologique, esthétique) qui ont voulu caractériser les modalités et les effets de l'acte de lecture. Don McKenzie partage un tel constat, mais il lui donne une dimension historique et dynamique en indiquant que « de nouveaux lecteurs créent des textes nouveaux dont les nouvelles signi-

8. Antoine Furetière, Dictionnaire universel, La Haye et Rotterdam, Arnout et Reinier Leers, t. III, 1690.

9. «Printed punctuation may reflect that of the author, that of the person who prepared the copy for the press, that of the compositorm or all three (Malcolm Parkes, Pause and Effect: An Introduction to the History of Punctuation in the West, Berkeley, University of California Press, 1993, p. 5). 
fications dépendent directement de leurs nouvelles formes ${ }^{10} »$. Est ainsi désignée avec acuité la relation qui lie la variation des formes dans lesquelles les œuvres sont données à lire, la composition de leurs publics dans ces différentes formes, et le sens que ceux-ci attribuent aux textes qu'ils font leurs.

L'étude morphologique des dispositions typographiques et l'analyse sociale des publics, loin de s'exclure, sont donc nécessairement associées. C'est en fonction des compétences et des attentes supposées des lecteurs visés par l'auteur, l'éditeur ou l'imprimeur que sont décidées les formes données aux textes. Mais celles-ci ont une dynamique propre qui peut, ou non, construire un nouveau public (par exemple plus large et plus populaire) et autoriser des appropriations inédites de textes qui, auparavant, ont circulé autrement et pour d'autres lecteurs. C'est grâce à la mobilité de leurs formes que les textes sont susceptibles de réemplois et de réinterprétations par les différents publics qu'ils atteignent, ou inventent. Mais pour autant ces formes typographiques contraignent-elles les lecteurs sans leur laisser diverses possibilités d'interprétation? Le soutenir serait déplacer sur la matérialité du texte, tenue pour impérieuse, le pouvoir que la critique structuraliste attribuait à la toute puissance linguistique du texte.

Pour éviter ce risque il est sans doute nécessaire de faire retour sur les propositions qui ont voulu donner à la lecture un rôle fondamental dans le processus de production du sens. Au sein de la critique littéraire des années soixante et soixante-dix du $\mathrm{Xx}^{\mathrm{e}}$ siècle le dilemme était le suivant: comment dans l'analyse libérer les lecteurs de la soumission obligée aux intentions de l'auteur ou à la machinerie linguistique du texte sans pour autant basculer dans un inventaire infini, disparate, de la diversité des lectures? Dans la perspective de l'esthétique de la réception, c'est le concept d'« horizon d'attente » qui doit permettre de surmonter la difficulté. Pour Hans Robert Jauss, tel qu'il le formule en 1970 dans son article «Literary History as a Challenge to Literary Theory ${ }^{11}$ ", il désigne les catégories esthétiques partagées auxquelles les œuvres généralement se conforment mais que les plus provocantes

10. D. F. McKenzie, op. cit., p. 53.

11. Hans Robert Jauss et Elizabeth Benzinger, «Literary History as a Challenge to Literary Theory» New Literary History, 2, 1, automne 1970, p. 7-37. 
d'entre elles (par exemple Don Quichotte ou Madame Bovary) transforment profondément. Pour lui,

l'analyse de l'expérience littéraire du lecteur échappera au psychologisme dont elle est menacée, si, pour décrire la réception de l'œuvre et l'effet produit par celle-ci, elle reconstitue l'horizon d'attente de son premier public, c'est-à-dire le système de références objectivement formulable qui, pour chaque œuvre au moment de l'histoire où elle apparaît, résulte de trois facteurs principaux : l'expérience préalable que le public a du genre dont elle relève, la forme et la thématique d'œuvres antérieures dont elle présuppose la connaissance, et l'opposition entre langage poétique et langage pratique, monde imaginaire et réalité quotidienne ${ }^{12}$.

La reconstitution des «horizons d'attente» des lecteurs doit ainsi rompre avec l'évidence d'un «sens objectif, une fois pour toutes arrêté, immédiatement accessible en tout temps à l'interprète ${ }^{13}$ ».

Confronté au même défi (comment construire la capacité inventive de la lecture sans tomber dans l'arbitraire des significations, aussi nombreuses et variés que les lecteurs?), Stanley Fish a proposé en 1980, dans son livre Is There a Text in this Class $?^{14}$, la notion d'«interpretive communities ». Destinée à éviter tant l'absolue tyrannie du texte que le radical solipsisme de la lecture, elle désigne les catégories interprétatives qui, pour n'être pas universellement partagées, ne sont pas non plus irréductiblement propres à chaque lecteur. Selon Fish, «l'acte qui fait reconnaître la littérature n'est pas contraint par quelque chose dans le texte, et il ne résulte pas non plus d'une volonté indépendante et arbitraire, il procède d'une décision collective à propos de ce qui doit être considéré comme littérature, une décision qui ne vaut qu’autant qu'une communauté de lecteurs continue à l'accepter ${ }^{15}$.» De là, la centralité de la notion de «communautés d'interprétation» qui doit

12. Hans Robert Jauss, Pour une esthétique de la réception, Paris, Gallimard, 1978, p. 49.

13. Ibid., p. $5^{8}$.

14. Stanley Fish, Is There a Text in this Class? The Authority of Interpretive Communities, Cambridge, Harvard University Press, 1980.

15. «Thus the act of recognizing literature is not constrained by some thing in the text, nor does it issue from an independant and arbitrary will ; rather, it proceeds, from a collective decision as to what will count as literature, a decision that will be in force only so long as a community of readers or believers continues to abide by it » (ibid., p. 11). 
permettre d'annuler la distance entre texte et lecteurs, entre objet et sujet, entre évidence du sens et expérience de lecture. Stanley Fish écrit: «Une communauté d'interprétation n'est pas objective parce que en tant qu'elle est définie par un ensemble d'intérêts, de propos et buts particuliers, sa perspective est engagée et non pas neutre, mais pour la même raison, les significations et les textes produits par une communauté d'interprétation ne sont pas subjectifs parce qu'ils ne résultent pas d'un individu isolé mais du point de vue d'un public partageant les mêmes ${ }^{16}$.» Pour lui, les stratégies d'interprétation qui définissent les différentes communautés de lecteurs sont, à la fois, décisives pour la construction du sens de ce qui est lu et antérieures à l'acte de lecture lui-même car elles impliquent les normes et valeurs qui gouvernent, pour chacune d'elles, leur relation à l'écriture.

On pourrait ranger aux côtés des notions proposées par Jauss et Fish la manière dont Barthes dans «La mort de l'auteur » (un essai d'abord publié en anglais dans Aspen Magazine en $1967^{17}$ ) définit la position de lecture comme le lieu où se construit un sens qui échappe au contrôle et la volonté de l'auteur: "un texte est fait d'écritures multiples, issues de plusieurs cultures et qui entrent les unes avec les autres en dialogue, en parodie, en contestation; mais il y a un lieu où cette multiplicité se rassemble, et ce lieu, ce n'est pas l'auteur, comme on l'a dit jusqu'à présent, c'est le lecteur: le lecteur est l'espace même où s'inscrivent, sans qu'aucune ne se perde, toutes les citations dont est faite une écriture; l'unité du texte n'est pas dans son origine mais dans sa destination ${ }^{18}$ ». D'où la conclusion sans appel: «la naissance du lecteur doit se payer de la mort de l'Auteur ${ }^{19}{ }^{\prime}$.

Ces trois perspectives ont eu le mérite d'«extraire» la lecture du texte, de lui assigner un rôle essentiel dans la construction du sens et de l'inscrire dans des conventions collectivement partagées. Leurs

16. «An interpretive community is not objective because as a bundle of interests, of particular purposes and goals, its perspective is interested rather than neutral: but by the very same reasoning, the meanings and texts produced by an interpretive community are not subjective because they do not proceed from an isolated individual but from a public and conventional point of view» (ibid., p. 14).

17. Roland Barthes, «The Death of the Author», trad. Richard Howard, Aspen, 5-6, 1967 .

18. Roland Barthes, "La mort de l'Auteur", dans Le bruissement de la langue: Essais critiques IV, Paris, Seuil, coll. «Points essais», 1984, p. 69.

19. Idem. 
limites, toutefois, étaient doubles. D’une part, elles considéraient les textes dans leur seul contenu linguistique ou sémantique, sans accorder aucune attention à leur matérialité même. D’autre part, de diverses façons, elles effaçaient les déterminations sociales et historiques de l'acte de lecture mis au cœur du processus de construction du sens. Barthes le fait en affirmant que «la destination [des textes] ne peut plus être personnelle: le lecteur est un homme sans histoire, sans biographie, sans psychologie; il est seulement ce quelqu'un qui tient rassemblé dans un même champ toutes les traces dont est constitué l'écrit ${ }^{20}$ », Jauss en supposant l'universalité, en un moment donné, «d'une expérience esthétique intersubjective préalable qui fonde toute compréhension individuelle d'un texte et l'effet qu'il produit ${ }^{21}{ }$ »et Fish en réduisant la définition des communautés de lecteurs à leurs seuls principes herméneutiques: «les communautés d'interprétation sont constituées par tous ceux qui partagent les mêmes stratégies d'interprétation $^{22}$ ».

Pour dépasser ces limites, il faut tenir le monde des textes comme un monde d'objets et de «performances» et le monde des lecteurs comme celui des communautés à laquelle ils appartiennent et que définissent un même ensemble de compétences, de pratiques et d'usages de l'écrit. Dans le prologue de l'édition de 1502 de la Tragicomédie de Calixte et Mélibée, mieux connue sous le titre de Celestina, Fernando de Rojas assigne les diverses interprétations de l'œuvre à la diversité des âges et des humeurs de ses auditeurs:

Les uns rongent des os qui n'ont point de vertu, soit l'histoire tout ensemble, et ne tirant profit de ses particularités, ils en font un conte pour voyage. Les autres piquent bons mots et proverbes communs, et, mettant attention à bien louer, ils oublient ce qui leur est plus utile et leur convient leur mieux. Mais ceux pour qui tout est plaisir véritable, ils rejettent l'anecdote bonne à conter, en retiennent la somme pour leur profit, rient aux joyeux propos et gardent en mémoire sentences et dits des philosophes pour les adapter au bon moment à leurs actes et à leurs desseins. Aussi, que dix personnes viennent à se réunir pour entendre cette comédie, en lesquelles il y a tant d'humeurs différentes comme il

20. Idem.

21. Hans Robert Jauss, op. cit., p. 50.

22. "Interpretive communities are made up of those who share interpretive strategies» (Stanley Fish, op. cit. p. 14). 
arrive toujours, niera-t-on qu'il n'y ait motifs de discussion sur des choses qui de tant de façons différentes se peuvent entendre ${ }^{23}$.

Près de cinq siècles plus tard, Borges attribue pareillement aux mutations des façons de lire les variations du sens des œuvres:

La littérature est chose inépuisable, pour la raison suffisante et simple qu'un seul livre l'est. Le livre n'est pas une entité close: c'est une relation, c'est un centre d'innombrables relations. Une littérature diffère d'une autre, postérieure ou antérieure à elle, moins par le texte que par la façon dont elle est lue : s'il m'était donné de lire n'importe quelle page d'aujourd'hui - celle-ci par exemple - comme on la lira en l'an 2000, je connaîtrai la littérature de l'an $2000^{24}$.

Avec de telles autorités, il n'est guère besoin de justifier les raisons qui ont inspiré la volonté de repérer «le lecteur à l'œuvre », lorsque s'opère la rencontre entre le monde du texte, qui est toujours un monde d'objets écrits ou de paroles lectrices ou déclamatrices, et un horizon d'attente, modelé par les conventions, les habitudes ou les expériences passées.

Mais saisir l'acte de lecture n'est pas chose aisée. Toutes les perspectives critiques qui l'ont placé au cœur de leur démarche, de l'esthétique de la réception à la «reader response theory », ont toujours oscillé entre, d'une part, la mise en évidence de catégories ou des stratégies partagées par tous les lecteurs et lectrices appartenant à une même communauté d'interprétation, au risque de manquer l'originalité de chaque appropriation, et, d'autre part, la fascination pour l'infinie diversité des interprétations et des usages des textes, au risque de tomber dans la collecte, elle-même infinie, des singularités. Comment transformer une telle tension en instrument de compréhension?

D'abord, en considérant la gamme très large de ce que, à chaque époque, lisent les lecteurs. La lecture n'est pas seulement lecture de livres, et moins encore uniquement lecture d'œuvres consacrées par le

23. Fernando de Rojas (y « antiguo autor »), La Celestina, Tragicomedia de Calisto y Melibea, éd. de Francisco J. Lobrera, Guillermo Serés, Paloma Díaz-Mas, Carlos Mota, Iñigo Ruiz Arzálluz et Francisco Rico, Barcelone, Cátedra, 2000, p. 20 (traduction française: La Célestine, ou Tragi-Comédie de Calixte et Mélibée, attribuée à Fernando de Rojas, traduction par Pierre Heugas, dans Théâtre espagnol du XVI e siècle, éd. de Robert Marrast, Paris, Gallimard, coll. «Bibliothèque de la Pléiade », 1983, p. 11).

24. Jorge Luis Borges, Enquêtes, Paris, Gallimard, coll. «Du monde entier », 1986 (1957), p. 208. 
canon littéraire ou philosophique. L'invention de l'imprimerie a certainement permis la dissémination des textes à une échelle inconnue au temps du manuscrit. Le constat ne fait pas débat. Avec l'invention de Gutenberg, plus de textes sont mis en circulation et chaque lecteur est à même d'en rencontrer un plus grand nombre. Mais quels sont ces textes dont la présence est démultipliée par l'imprimerie? Des livres, bien sûr, mais, comme l'a montré D. F. McKenzie, leur impression constitue une part souvent minoritaire, voire très minoritaire de l'activité des ateliers typographiques entre le $\mathrm{XV}^{\mathrm{e}}$ et le XvIII ${ }^{\mathrm{e}}$ siècle. L'essentiel de la production imprimée consiste en libelles, pamphlets, pétitions, affiches, formulaires, billets, quittances, certificats, et bien d'autres «travaux de ville » qui assurent le plus clair des revenus des entreprises. L'imprimerie offre ainsi à la lecture des objets inconnus ou marginaux à l'âge du manuscrit et dans les villes de la première modernité, l'écrit imprimé s'empare des murs, se donne à lire dans les espaces publics, transforme les pratiques administratives et commerciales.

Pour autant, l'écrit imprimé ne règne pas en maître absolu et exclusif. Après les travaux consacrés à la publication manuscrite en Angleterre, en Espagne, et en France, il n'est personne aujourd'hui pour soutenir que «ceci» (la presse à imprimer) a tué «cela» (le manuscrit). Entre le $\mathrm{XVI}^{\mathrm{e}}$ et le $\mathrm{XVIII}{ }^{\mathrm{e}}$ siècle, multiples sont les genres (anthologies poétiques, libelles politiques, instructions nobiliaires, nouvelles à la main, textes libertins et hétérodoxes, écrits matérialistes, partitions musicales, etc.) qui furent très souvent lus dans des copies manuscrites, et non pas des ouvrages imprimés. Les raisons en sont nombreuses: le moindre coût de l'«édition », la volonté de déjouer la censure, le désir d'une circulation restreinte, ou encore, la malléabilité de la forme manuscrite, qui permet additions et révisions. Limprimerie, du moins dans les quatre premiers siècles de son existence, n’a fait disparaître ni la communication ni la publication manuscrites.

Plus encore, elle a invité à de nouveaux usages de l'écriture à la main comme l'atteste un premier inventaire des objets qui incitent leurs acheteurs à remplir avec leur écriture les espaces que l'impression a laissés en blanc. Il en va ainsi des pages vierges interfoliées dans les almanachs, des espaces en attente d'écriture dans les formulaires ou des larges marges et interlignes des ouvrages destinés à accueillir les annotations du lecteur. Il serait aisé de multiplier les exemples de ces objets imprimés dont la raison d'être est de susciter et préserver l'écri- 
ture manuscrite: ainsi, les éditions des classiques latins utilisées dans les collèges, les chartes de mariage, en usage dans certains diocèses de la France méridionale au XVII ${ }^{\text {e }}$ siècle, ou, au siècle suivant et en Italie, les premiers agendas dans lesquels chaque jour est divisé en ses différents moments. Les proximités entre écritures manuscrites et textes imprimés ne sont d'ailleurs pas limitées à ces seuls objets qui, explicitement, les organisent. Les lecteurs du passé, en particulier les lecteurs lettrés, se sont souvent emparés des ouvrages sortis des presses en corrigeant à la plume les erreurs qu'ils y trouvaient et en établissant les index ou les erratas manuscrits qui leur étaient utiles, voire en composant des livres originaux à partir des fragments d'éditions imprimés qu'ils découpaient et collaient.

Les spectaculaires exemples d'annotations manuscrites collectées par les historiens peuvent conduire à faire retour sur l'historicité des pratiques qui accompagnent les lectures. À la Renaissance, annoter un livre avec des soulignements, des rubriques marginales ou des références croisées est l'un des gestes imposés par la technique des lieux communs. Ainsi repérés et désignés, les passages qui importent sont ensuite copiés et distribués entre les rubriques thématiques d'un cahier ou livre de lieux communs. Procurant des faits attestés par les Anciens ou les contemporains, des modèles de style et d'argumentation ou des sentences, qui sont autant de vérités universelles, donc «sublimes" pour reprendre le mot de Francis Goyet, ces lieux communs peuvent venir nourrir les discours de celui qui les a rassemblés. Les imprimeurs s'emparent de la technique en publiant des livres en blanc où seules sont imprimées les titres des rubriques ou bien en proposant des anthologies où sont déjà compilées et organisées les citations ou paraphrases dont les lecteurs pourraient avoir besoin.

La pratique s'empare de tous les genres et aura une longue vie. Au XVII ${ }^{\mathrm{e}}$ siècle, elle transforme les répliques du théâtre en maximes générales. En rassemblant trente-six de ses pièces, le Folio de 1623 inaugure la canonisation de Shakespeare. Mais c'est dès 1600 que des citations de ses poèmes, le Viol de Lucrèce et Vénus et Adonis, et de cinq de ses pièces apparaissent dans des recueils de lieux communs, entièrement composés à partir d'auteurs qui ont écrit et écrivent en anglais, et non en latin: d'une part, le Bel-vedere, or The Garden of the Muses, d'autre part, England's Parnassus. Plus tard, en 1751 et 1755 , c'est Richardson lui-même qui compile dans ses propres romans des 
aphorismes, sentiments, réflexions et observations qui constituent, rangés sous des rubriques données en ordre alphabétique, des exemples «digested under proper heads», ce qui est reprendre le lexique ancien, médiéval et renaissant, biblique et humaniste, qui décrit la lecture comme une «ruminatio » et «digestio ».

En ce sens, Montaigne qui ne tient pas de «gardoires» ou cahiers de lieux communs, qui copie directement dans ses Essais des extraits (parfois fort longs) d'autres livres et qui écrit dans le livre lui-même «l'idée générale» qu'il en a retenu n'est pas un praticien exemplaire de la technique intellectuelle dominante de son temps. Inversement, les lecteurs du XVIII ${ }^{\mathrm{e}}$ siècle ne sont pas tous des Richardson. Leurs annotations marginales se sont libérées des obligations des lieux communs et utilisent les blancs de la composition pour manifester leur réaction face à l'œuvre et pour se l'approprier tant dans son existence matérielle d'objet acheté, offert, reçu, dont les pérégrinations sont rappelées sur la page de titre, que dans son texte lui-même, qui suscite émotions, souvenirs, et désirs. Les «marginalia», qui sont une manne pour les historiens de la lecture comme pour ceux des œuvres, ont donc une réalité morphologique de longue durée puisque, dans le manuscrit médiéval comme dans le livre imprimé d'aujourd'hui, elles doivent trouver leur place dans un objet qui, sauf exception, ne les attend pas. Mais ces annotations ont aussi une histoire et elles s'inscrivent dans des pratiques intellectuelles ou des sensibilités qui ne sont pas des invariants.

Durablement, l'écrit a été tenu pour l'instrument de pouvoirs redoutables et redoutés. Caliban le sait, qui pense que la puissance de Prospero sera détruite si ses livres sont saisis et brûlés: «Burn but his books ${ }^{25}$ ». Mais les livres de Prospero ne sont en fait qu'un seul livre: celui qui lui permet de soumettre à ses volontés la Nature et les êtres. Ce pouvoir démiurgique est une terrible menace pour qui l'exerce, et copier ne suffit pas toujours à conjurer le péril. Le livre doit disparaître, noyé au fond des eaux: «Et plus profond que jamais n'est descendue la sonde, / Je noierai mon livre [I'll drown my book $\left.{ }^{26}\right]$ ». Trois siècles plus tard, avec Borges, c'est dans d'autres profondeurs, celles des magasins de la bibliothèque nationale de la rue México, que doit être enseveli un livre qui, pour être de sable, n’en était pas moins inquiétant.

25. Shakespeare, The Tempest, III, ii.

26. Ibid., V, i. 
Au XVIII siècle, les corps eux-mêmes indiquent, pour le pire ou parfois le meilleur, les dangers ou les bienfaits de la lecture. Le discours se médicalise, construisant une pathologie de l'excès de lecture considéré comme une maladie individuelle ou une épidémie collective. La lecture sans contrôle est tenue pour dangereuse parce qu'elle associe l'immobilité du corps et l'excitation de l'imagination. Elle entraîne, de ce fait, les pires maux: l'engorgement de l'estomac et des intestins, le dérangement des nerfs, l'épuisement du corps. Comme l'indique le docteur Tissot, les professionnels de la lecture, à savoir les hommes de lettres, sont les plus exposés à de tels dérèglements, sources de la maladie qui est par excellence la leur: l'hypocondrie. D'autre part, l'exercice solitaire de la lecture conduit à un dévoiement de l'imagination, au refus de la réalité, à la préférence donnée à la chimère. De là, la proximité entre l'excès de lecture et les plaisirs solitaires. Les deux pratiques produisent les mêmes symptômes: la pâleur, l'inquiétude, la prostration. Le danger est maximal quand la lecture est lecture d'un roman libertin ou érotique et le lecteur une lectrice retirée dans la solitude. La lecture est donc désormais pensée à partir de ses effets corporels et cette somatisation d'une pratique, dont les dangers étaient traditionnellement désignés à l'aide de catégories philosophiques ou morales, comme dans le néo-platonisme de la Renaissance, est peut-être le premier signe d'une forte mutation des comportements et des représentations.

Mais le corps peut aussi révéler l'émotion la plus sincère, celle produite par l'identification à un texte qui procure une connaissance pragmatique des choses et des êtres et fait intérioriser, dans l'évidence du sentiment, le partage entre le bien et le mal. C'est un tel bouleversement des sens que produit, pour Diderot, la lecture de Richardson. Il décrit ainsi son émoi à la lecture du récit de l'enterrement de Clarissa dans une lettre à Sophie Volland du 17 septembre 1761: «Seulement encore mes yeux se remplirent de larmes; je ne pouvais plus lire; je me levai et me mis à me désoler, à apostropher le frère, la sœur, le père, la mère et les oncles, et à parler tout haut, au grand étonnement de Damilaville qui n'entendait rien ni à mon transport ni à mes discours, et qui me demandait à qui j’en avais ${ }^{27}$.» Quelques mois plus tard, dans

27. Denis Diderot, Correspondance, éd. de Laurent Versini, Paris, Robert Laffont, coll. «Bouquins», 1999, p. $34^{8}$. 
l'Éloge de Richardson qu'il rédige pour le Journal étranger, c'est à ce même Damilaville qu'il attribue les réactions qui avaient été les siennes:

J'étais avec un ami, lorsqu'on me remit l'enterrement et le testament de Clarisse, deux morceaux que le traducteur français a supprimés, sans qu'on sache trop pourquoi. Cet ami est un des hommes les plus sensibles que je connaisse et un des plus ardents fanatiques de Richardson : peu s'en faut qu'il ne le soit autant que moi. Le voilà qui s'empare des cahiers, qui se retire dans un coin et qui lit. Je l'examinais: d'abord je vois couler des pleurs, bientôt il s'interrompt, il sanglote; tout à coup il se lève, il marche sans savoir où il va, il pousse des cris comme un homme désolé et il adresse les reproches les plus amers à toute la famille des Harloves ${ }^{28}$.

Des mouvements du corps et de l'âme toujours plus violents scandent l'irrépressible bouleversement qui envahit le lecteur, les pleurs, les sanglots, l'agitation, les cris et, finalement, les imprécations, manifestant ainsi, selon la belle formule de Jean Starobinski, que «l'énergie dont le roman est la source peut être intégralement reversée sur la vie réelle ${ }^{29} »$.

Pourtant tous les lecteurs de Richardson ne partagent pas nécessairement l'émoi qui saisit Damilaville ou Diderot. Celui-ci met en scène dans son Eloge de Richardson une méchante lectrice qui rit là ou d'autres pleurent et demeure insensible devant les malheurs de Clarisse. La petite fiction de la lectrice indifférente a peut-être été construite par Diderot à partir d'une lettre authentique, soit de Sophie Volland évoquant sa mère, soit, plus sûrement, de Madame d'Épinay. Elle indique, d'abord, qu'entrer dans la communauté des lecteurs de Richardson n'est pas donné à tous - ou à toutes. Seuls les êtres sensibles et bons, qui reconnaissent leurs semblables dans les personnages vertueux des romans, composent cette société harmonieuse où les cœurs sont en correspondance et où chacun devient meilleur. Mais elle dit aussi autre chose : le possible dévoiement de la lecture, l'appropriation sauvage et déréglée du texte. Les contraintes, explicites ou cachées, textuelles ou matérielles, des discours ne les préservent pas des détour-

28. Denis Diderot, Contes et romans, éd. de Michel Delon, Paris, Gallimard, 2004, p. 908.

29. Jean Starobinski, "Se mettre à la place": la mutation de la critique de l'âge classique à Diderot », Cahiers Vilfredo Pareto, 14, 1976, p. 377. 
nements non voulus. L'histoire des lectures doit faire son profit du constat de tels écarts et considérer que si le lecteur est un effet du texte, il en est aussi le créateur.

Entre les machineries des discours et les attentes ou appropriations des lecteurs, la correspondance n'est pas obligée. De ces discordances, le XVIII ${ }^{\mathrm{e}}$ siècle est sans doute un exemple pertinent. D'un côté, il appartient pleinement à l'ancien régime typographique. Comme aux siècles précédents et dans toute l'Europe, la publication des livres est caractérisée par l'existence d'une censure préalable (sauf en Angleterre), la domination des libraires sur l'activité éditoriale et la stabilité du travail dans l'atelier d'imprimerie, qui depuis le $\mathrm{Xv}^{\mathrm{e}}$ siècle impose la limitation des tirages, permet les corrections sous presse et attribue un rôle essentiel aux correcteurs et compositeurs dans la fabrique du livre. En ce sens, tout typographique, le XVIII ${ }^{\mathrm{e}}$ siècle est plus proche du temps des incunables que celui de la double révolution, de l'impression puis de la composition, qui transforment profondément ateliers et édition au XIX' siècle.

D'un autre côté, c'est au sein de ces fortes continuités que naissent de nouvelles relations à la culture écrite qui sont déjà celles de la modernité. Dans toute l'Europe des Lumières, la croissance de l'offre imprimée, la circulation des livres interdits, la multiplication des périodiques, le triomphe des petits formats et l'essaimage des cabinets littéraires et des sociétés de lecture où il est possible de lire sans acheter, permettent et imposent de nouvelles manières de lire. Les unes consomment avec avidité et rapidité les imprimés nombreux, nouveaux et souvent éphémères, proposés par le commerce de librairie. Le regard du lecteur devient plus distancié et plus critique. À une relation à l'écrit communautaire et respectueuse s'oppose ainsi une lecture faite d'irrévérence et de désinvolture. Mais d'autres pratiques imposent, elles, des gestes tout différents, voire contraires. Le roman tel que le réinventent Richardson puis Rousseau et Goethe s'empare de son lecteur, le capture, gouverne ses pensées et ses conduites. Il est lu et relu, connu, cité, récité. Son lecteur est envahi par un texte qui l'habite et, en s'identifiant aux héros de l'histoire, c'est sa propre existence qu'il déchiffre dans le miroir de la fiction. Dans cette lecture particulièrement intense, la sensibilité tout entière se trouve engagée.

La coexistence de ces deux styles de lecture, au moins dans les milieux sociaux favorisés, tout comme les lectures contradictoires des 
mêmes textes qu'ils permettent, interdisent d'affirmer - ou récuser trop hâtivement la réalité d'une «révolution» de la lecture au $\mathrm{XVIII}^{\mathrm{e}}$ siècle. D'ailleurs, l'essentiel n'est peut-être pas là, mais bien plutôt dans le constat qui établit que, si les lectures ne peuvent pas être déduites des textes ou des livres et le lecteur identifié avec celui attendu par les auteurs ou les éditeurs, elles sont néanmoins situées dans un ensemble de conditions de possibilité définies dans chaque moment historique par les stratégies d'écriture, la matérialité même des écrits et les horizons d'attente des communautés d'interprétation. Comprendre comment, en des temps et des lieux différents, pour des genres et des publics divers, se croisent ces contraintes imposées et les libertés qu'elles permettent ou proposent est sans doute la tâche qui nous réunit. 